\title{
Design and Performance Analysis of Contra-rotating Pumpjet Propulsor under Condition of Torque Unbalance
}

\author{
Xiao-er Wang ${ }^{1, a}$, Zhen-shan Zhang ${ }^{1}$ and Meng Zhang ${ }^{1}$ \\ ${ }^{1}$ Naval University of Engineering Weaponry Engineering Department, 430033Wuhan, China
}

\begin{abstract}
In order to balance the torque of front rotor and rear rotor of underwater vehicle, the analysis of the speed triangles at the inlet and outlet of the front and rear rotor has been done. Then, the thought of using contra-rotating pumpjet to achieve the objective was raised. The stator is installed behind the rear rotor so as to ensure the overall torque of the propulsor balance, at the same time, the stator can also support the shroud of the propulsor. the parameter design of the rotor and the stator has been carried out by using the three dimensional inverse design method. At last, the performance of the designed pumpjet propulsor is obtained when it is installed on the underwater vehicle By using computational fluid dynamics. The results show that the total torque of the propulsor is reduced to $1.8 \mathrm{~N} * \mathrm{~m}$ on the design point although the power difference ratio of the front rotor and the rear rotor is $20 \%$. The torque ratio is also reduced from $4.6 \%$ to $0.4 \%$, which is good to meet the propulsor balance requirement and verifies the 3-D design method of pumpjet is effective.
\end{abstract}

\section{1 introduction}

As the speed of underwater vehicle which propelled by counter-rotating propeller increasing, the cavitation incipient of the front and the rear propeller has begun to become a serious problem. The generation and collapse of the cavitation bubble at high speed will increase the noise sharply which of course will reduce the concealment of the vehicle. once the cavitation is serious, the propeller may even facing the danger of stall. On the other hand, the increase of the speed will increase the resistance of the vehicle so that the power of the engine will be bigger than before. In order to ensure the operation of the engine, it is common to use part of the power of the front shaft for the auxiliary engine of the vehicle, which makes the power received by the front and rear rotor no longer equal. When the engine power is increased, the difference between the power of the front and rear rotor will be further increased when compared with the low speed, so the design of contra-rotating propeller becomes more difficult under the condition of unbalanced shaft power.

In order to reduce the propeller noise and improve the speed of the vehicle, a counter-rotating pump jet propulsor has been designed which used on the "stingray-1" torpedo[1]. Some research on the Contra-Rotating axial flow pump has been done in Japan[2]. But it is needed on the design method and performance analysis of the contra-rotating pumpjet under the condition of unbalanced shaft power.

\footnotetext{
${ }^{a}$ Xiao-er Wang : hnwzwxe@163.com
} 
Based on the three dimensional flow theory, the flow field calculation is completed after the iterative calculation between S1 flow surface and S2 flow surface is used to solve the continuous equation and the equation of motion in the pumpjet, then The blade shape is obtained by substituting the flow field calculation results into the blade equation. So that a new flow field will be calculated after the blades are obtained. The final blades can be obtained if the errors of the previous flow field and the last flow field meet the requirement. assumed that the are equal between the front and rear rotor, then the blades of the pumpjet propulsor will be obtained. Then, By means of computational fluid dynamics (CFD), numerical experiments were carried out on the designed pumpjet propulsor, the performance and the condition of torque balance for the propulsor under design speed were also can be analysed.

\section{Speed triangles analysis}

Assuming that the direction of the incoming flow to the pumpjet is axial, the velocity components inside the propulsor are analyzed as shown in Figure 1 (where subscript 1 indicates the inlet and subscript 2 indicates the outlet; The subscript $f$ indicates the front impeller, the subscript $r$ indicates the rear impeller). The rear rotor speed is 1.1 times the front rotor, But rotation direction in the opposite direction. Assuming that the flow field keeping same between the front rotor outlet and inlet of the rear rotor. The same applies to the rear rotor and the stator. So,

$$
V_{m 2 f}=V_{m 1 r} \quad V_{m 2 r}=V_{1 s}
$$

$V_{w 1 f}$ and $V_{w 2 f}$ respectively represent the front rotor inlet and outlet relative speed. $V_{w 1 r}$ and $V_{w 2 r}$ respectively represent the rear rotor inlet and outlet relative speed. $V_{1 s u}$ and $V_{1 s m}$ Represents the velocity component of $V_{1 s}$ in the circumferential direction and the velocity component of $V_{1 s}$ in the axial direction. $V_{2 s}$ represents the final outflow velocity. As shown in Figure 1, because the tail of vehicle is conical, the circumferential velocity at the inlet of the front and the rear rotor is lower than the circumferential velocity at the outlet.

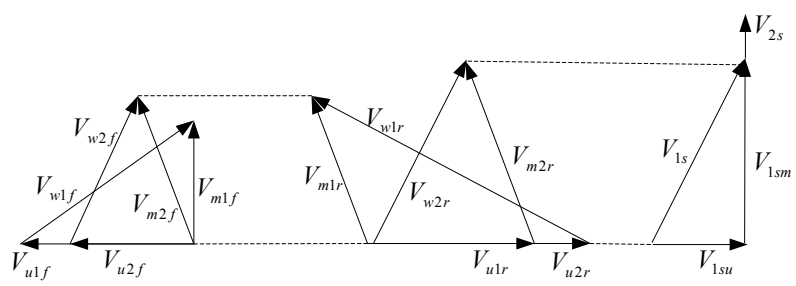

Figure 1. Inlet and outlet velocity triangles of contra-rotating pumpjet

\section{Basic equations}

Assuming that the flow of water in the pumpjet is steady, viscous and incompressible, the rotor comes in a non-swirling flow. Regardless of the thickness of the blade, the center plane of the blade is considered as a vortex sheet, and the vortex on the central plane is used to replace the effect of the blade on water. The strength of the vortex is controlled by the average circumferential circulation $2 \pi r \bar{V}_{\theta}[3-4]$.

$$
r \bar{V}_{\theta}=\frac{B}{2 \pi} \int_{0}^{\frac{2 \pi}{B}} r V_{\theta} d \theta
$$

Where, B represents the number of blades, $r$ represents radius, $V_{\theta}$ represents circumferential velocity.

Before the blade shape obtained, It needs to solve the following three equations based on S2 flow surface theory [3]:

(1) Continuity equation: 


$$
\frac{\partial}{\partial z}\left(H_{2} H_{3} \bar{V}_{z}\right)+\frac{\partial}{\partial r}\left(H_{1} H_{3} \bar{V}_{r}\right)=0
$$

Where, $H_{1}, H_{2}, H_{3}$ are the Lame coefficient in the curvilinear coordinate system, which are taken as 1 , $1, \mathrm{r}$, respectively in this paper. $\bar{V}_{r}$ and $\bar{V}_{z}$ represents the radial average velocity and the axial average velocity, respectively.

(2) Motion equation

$$
\begin{aligned}
F(r, z)=-\frac{\partial \bar{V}_{\theta} r}{\partial r} & \frac{\partial \varphi_{m}}{\partial z}+\frac{\partial \bar{V}_{\theta} r}{\partial z} \frac{\partial \varphi_{m}}{\partial r} \\
+ & \frac{1}{w^{2}}\left[\frac{\partial \bar{E}_{r}}{\partial r}\left(\bar{w}_{z}+\bar{w}_{\theta} \frac{r \partial \varphi_{m}}{\partial z}\right)-\frac{\partial \bar{E}_{r}}{\partial z}\left(\bar{w}_{z}+\bar{w}_{\theta} \frac{r \partial \varphi_{m}}{\partial r}\right)\right]
\end{aligned}
$$

where, $\varphi_{m}$ is the average circumferential S2 flow surface equation. represents the energy of unit mass of water at the inlet of the pumpjet propulsor[4].

(3) Blade equation

$$
\bar{V}_{\theta}-\omega r=\frac{H_{3} \partial \varphi_{m}}{H_{1} \partial z} \bar{V}_{z}+\frac{H_{3} \partial \varphi_{m}}{H_{2} \partial r} \bar{V}_{r}
$$

Where, $\omega$ represents the rotor angular velocity.

Formulas(3), (4) and (6) form the system equations used to obtain the flow field and blade. It can be seen that $\bar{V}_{z}, \bar{V}_{r}, \bar{V}_{\theta}$ and $r \bar{V}_{\theta}$ are unknowns in the system of equations. In order to close the system, the distribution of the $r \bar{V}_{\theta}$ along the meridian streamlines is required during the design process.

According to some test data, $\bar{E}_{r}$ distribution at the inlet can be obtained. As the viscosity of water in the pumpjet is not considered, the $\bar{E}_{r}$ along the streamline is constant. Streamlines will be determined by iterative calculations. The steps are as follows: (1) Given the meridian surface of the pumpjet, the position of leading edge and trailing edge of the rotor and stator. Angular velocity and other boundary conditions are also needed; (2) the initial streamline and velocity field can be obtained by solved equation (4) when its right side assuming to equal to zero; (3) Method of Characteristics will be used to solve the equation (6), in order to obtain the $\varphi_{m}$. (4) The distribution of $F(r, z)$ obtained from equation (5) and then equation (4) will be calculated when the result of $F(r, z)$ is Substituted in (4); (5) Repeat step (4) and step (5) until the error meets the requirement ; (6) Blades of pumpjet will be obtained after thickened according to the NACA data.

\section{Pumpjet design}

Before the design of pumpjet, the following parameters should be known. Rotating speed of the front rotor and the rear rotor, flow rate $Q_{d}$, the distribution of $\partial r \bar{V}_{\theta} / \partial m$, the number of front rotor, rear rotor and stator. In order to meet the shaft power of the front rotor and the rear rotor at the same time, keep the shroud of pumpjet taper, the rotating speed of rear rotor is slightly higher than the front rotor.

\subsection{Meridional geometry of the pumpjet}

Meridional shape of the pumpjet is closely related to the flow rate, efficiency, cavitation performance, so it needs to be determined according to the past experience, thrust requirement, and the geometry 
constraint of the underwater vehicle body. In this paper, Meridional shape of the pumpjet is plotted according to the actual size constraints of the tail of the navigating body and the required axial thrust force at the designed speed. The meridional geometry of the pumpjet is shown in Fig. 2.

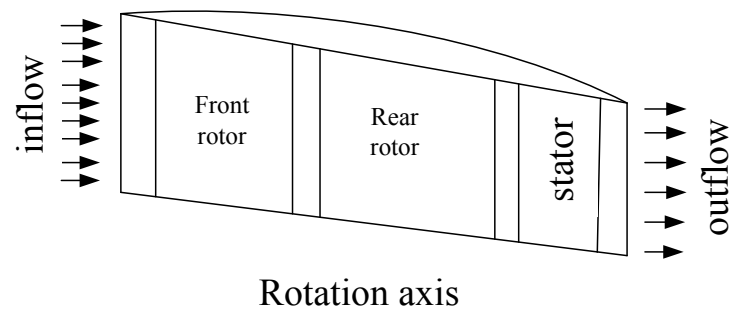

Figure 2. Meridional geometry of the pumpjet

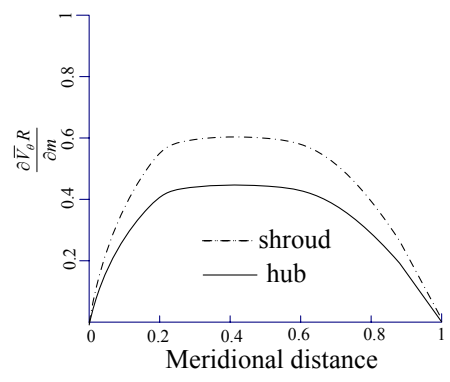

Figure 3. Blade loading distribution of the front rotor

\subsection{Loading distribution of Pumpjet}

Distribution of $\bar{V}_{\theta} r$, also known as loading distribution (the pressure difference between the blade pressure surface and suction surface), which has a significant impact on the efficiency and cavitation, so choose a reasonable loading distribution is key to design the blade of pumpjet.

According to Eq.(6), it can be seen that the loading on the blade is related to the partial derivative of $\bar{V}_{\theta} r$. Therefore, in order to facilitate the iterative calculation, the distribution of $\bar{V}_{\theta} r$ along the streamline line is given here. This method is effective to precise control of the loading, which makes low pressure zones can be prevented [5-6]. At the same time, the blade loading distribution needs to satisfy the following two conditions [7]:

(1) At the Exit,

$$
\frac{\partial r \bar{V}_{\theta}}{\partial m}=0
$$

This is because the pressure at the pressure surface should be equal to the pressure at the suction surface according to the kutta condition.

(2) Integral value from the leading edge to the trailing edge is equal to the circulation at the trailing edge, namely:

$$
\int_{0}^{1} \frac{\partial r \bar{V}_{\theta}}{\partial m} d m=V_{\theta_{2}} r_{2}
$$

where, $m=0$ represents blade leading edge. $m=1$ represents blade trailing edge, the subscript 2 represents the value at the outlet, and $V_{\theta 2} r_{2}$ is the speed moment at the outlet.

According to reference [5-6], we decided to take into account both the efficiency and cavitation of the pumpjet propulsor, using the intermediate uniform loading distribution form, that is, the maximum load appeared at 0.2 chord length, then keep it unchanged and gradually decreased at 0.6 chord length. The loading distribution at the hub and shroud of the front rotor and the rear rotor are showed in figure 3 and figure 4 . The loads at the other radius cross sections are obtained by linear interpolation.

One of the roles of the stator is to convert the rotating flow discharged from the rotor into an axial flow to improve the efficiency. On the other hand it can plays the role of balance the propulsor's torque and support the shroud. In this paper, the main research about the stator is focus on whether the outflow from the pumpjet is straight, while avoiding the secondary flow. The stator loading distribution is based on the reference [7], as shown in Fig. 5, the loading distribution at the hub and the shroud is given, and the other radius sections obtained by linear interpolation. 


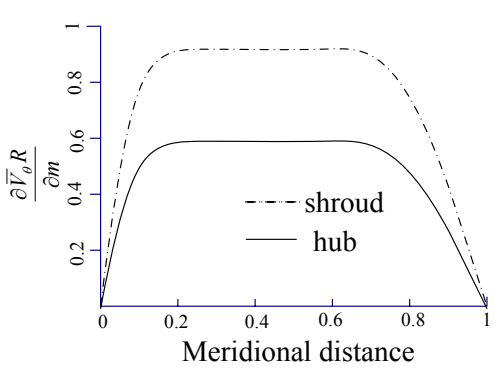

Figure 4. Blade loading distribution of the rear stator stator

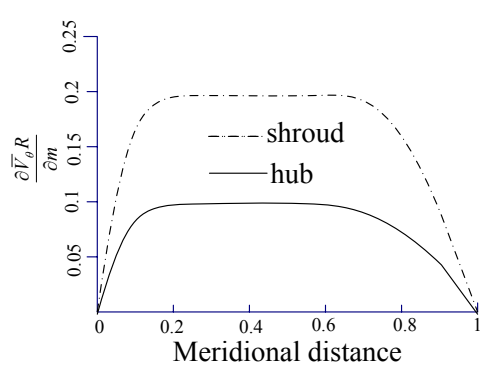

Figure 5. Blade loading distribution of the

\section{3 blade thickening}

After obtaining the blade without thickness, The final pumpjet geometry will be obtained through thickening the blade with NACA series airfoil thickness distribution rule.

\section{Pumpjet propulsor performance caculation}

After obtaining the geometry of the pumpjet, the pumpjet propulsor is placed on the tail of the underwater vehicle. Then, the numerical self-propelled calculation result can be analyzed.

\subsection{Numerical model}

In this paper, the central node control and finite volume method are used to solve the RANS equation. SST turbulence model to complete the system closed [8].

\subsection{Mesh and boundary conditions settings}

After obtaining the front rotor, the rear rotor and the stator of the pumpjet propulsor, the rotor mesh is completed by Turbo-Grid. J-type topology is selected in the process of generating mesh of the front and the rear rotor, and the tip clearance is taken as $0.5 \mathrm{~mm}$. The grids are selected as 376,000 and 371,000 . The stator selects $\mathrm{H}$ type topological structure, the quantity of the grid is chosen as 218,000 . The computational domain size is selected according to reference [8], grids number of computational domain is 7.28 million. Boundary conditions are set to speed inlet and pressure outlet. The front and rear rotors and their hubs are set at relatively stationary wall boundary and their shrouds are set to absolute stationary wall boundary [8]. The interface between the calculation domain and the front rotor, the interface between the front rotor and the rear rotor and between the rear rotor and the stator are set as the frozen rotor mode.

\subsection{Calculation results}

The result of self-propelled of the fully attached underwater vehicle is shown in Fig.6. It can be seen that at design speed, the thrust of pumpjet is slightly larger than the resistance of vehicle. After the speed increases slightly, the thrust and resistance are basically balanced. The post-treatment shows that the thrust efficiency of the pumpjet propulsor at this time is $67.9 \%$, which basically meets requirements. Under the design speed, the pumpjet has reduced the overall imbalance moment of the propulsor to $1.8 \mathrm{~N} * \mathrm{~m}$ by appropriately increasing the speed of the rear rotor and the balance of the stator under the condition that the front and the rear rotor have a power difference of $20 \%$, The proportion of the total torque was reduced from $4.6 \%$ to $0.4 \%$, basically completing the design goal of overall moment balance, which verified the validity of the design ideas and methods. The reference [2] 
shows that the efficiency of the pumpjet is lower under the condition of the rear rotor has a higher rotary speed than that the front and the rear rotors have a same rotary speed.

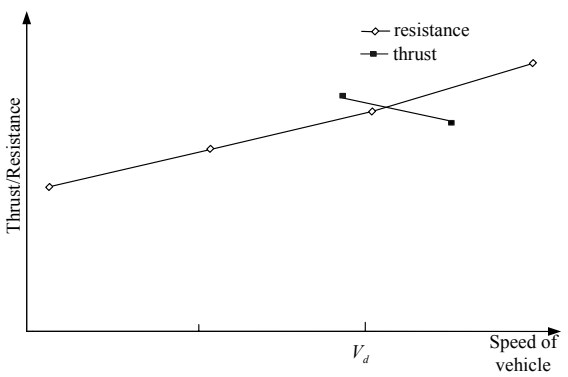

Figure 6. Speed evaluation of underwater vehicle

The pressure distribution of the pumpjet blade under the condition of the design speed is shown in Fig.7 and Fig.8. It can be seen from the figure that except for the leading edge, in which area the blades have a violent action with water, the pressure distribution of the pressure surface and the suction surface is smooth. There is no outstanding pressure fluctuation, which shows that the blade loading distribution is uniform. there is no pressure gradient larger area, to avoid the local low pressure area, reducing the risk of cavitation. This is also best demonstrated by the absence of a blue depression in each of the blades of the pressure contour in Figure 9.

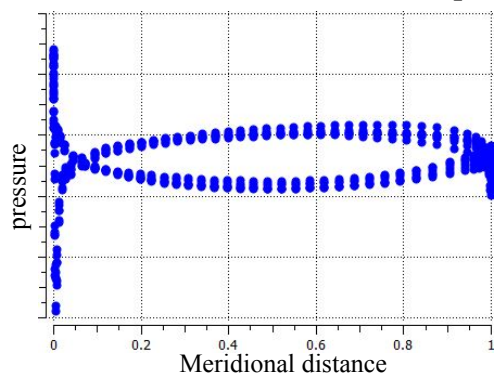

Figure 7. Pressure loading distribution of the front rotor rear rotor

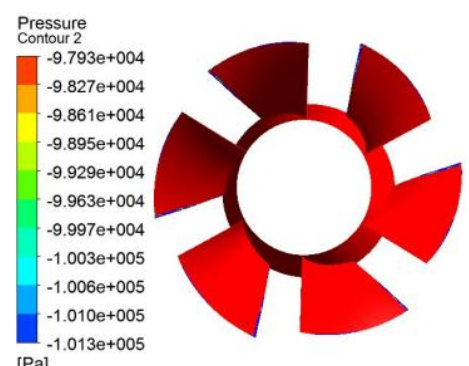

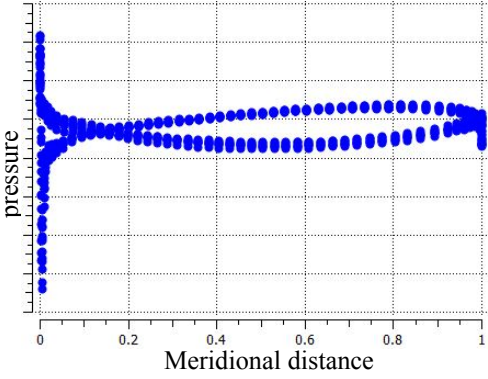

Figure 8. Pressure loading distribution of the

Figure 9. Pressure contour of rotor and stator

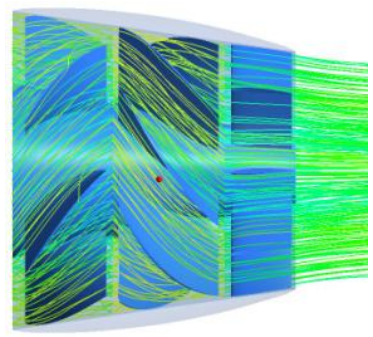

Figure 10. Three-dimensional streamline of the pumpjet 
As shown in Fig.10, from the pumpjet internal flow chart, it can be seen that the entire flow is smooth without disorder. The flow around the pumpjet rotor and the stator blades at three different radius is observed. The vortex flow between the blades is not seen in Fig.11, and the final flow is smooth and straight, which indicates that the design of the stator is good.

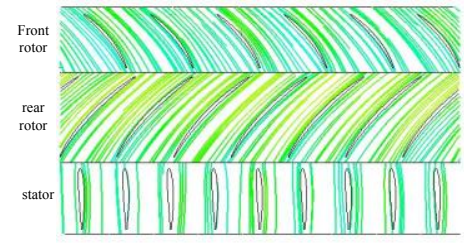

(a) $0.3 \mathrm{R}$ sectional drawing

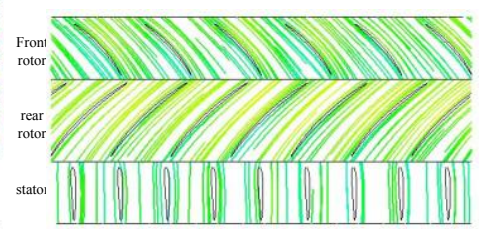

(b) $0.5 \mathrm{R}$ sectional drawing

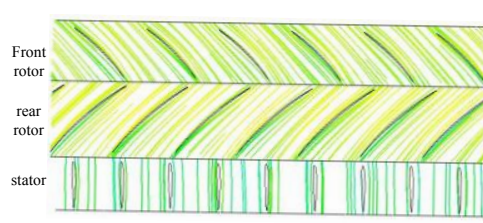

(c) $0.9 \mathrm{R}$ sectional drawing

Figure 11. Two-dimensional streamline at the different radius of the pumpjet

\section{Conclusion}

(1) A pumpjet of underwater vehicle was designed using the three dimensional inverse method after analyzing the speed triangle of the pumpjet. The design process has less assumptions about the internal flow of the pumpjet.

(2) The $r \bar{V}_{\theta}$ is used to control the loading of the blade, which well balances the entire propulsive torque. Meanwhile, the distribution of the velocity moment effectively controls the pumpjet efficiency and the cavitation of the blade. The method is simple and effective. The loading distribution, which selected according to the reference [8], effectively takes into account the efficiency and anti-cavitation performance of the pumpjet, and there is no obvious low pressure area on the rotor surface.

(3) Under the design speed, the thrust the pumpjet and the resistance of the vehicle is nearly balanced, and the thrust efficiency reached $68 \%$, which verifies the thought of the pumpjet's structure is useful.

\section{References}

1. Wang Lan, The history of torpedo development in the world and its landmark study[J]. Underwater weapons, 2:1( 2015)

2. A. Furukawa, Y. Cao, K. Okuma, S. Watanabe, Experimental Study of Pump Characteristics of Contra-Rotating Axial Flow Pump, Proc. 2nd International Symposium on Fluid Machinery and Fluid Engineering, Beijing, China,245(2000)

3. Luo Xingqi. Full three-dimensional inverse and optimum design of Francis runner [D].Beijing: Tsinghua University(1995)

4. Peng Guoyi. Three-dimensional rotational flow inverse computation and optimum design of Kaplan runner[D]. Beijing: Tsinghua University(1996)

5. Duccio Bonaiuti, Mehrdad Zangeneh. Parametric Design of a Waterjet Pump by Means of Inverse Design, CFD Calculations and Experimental Analyses[J].ASME Journal of Fluids Engineering, 132,1(2010)

6. Duccio Bonaiuti, Mehrdad Zangeneh. On the coupling of inverse design and optimization techniques for the multi-objective, multipoint design of turbomachinery blades[J].ASME Journal of Turbomachinery, 131,1014(2009)

7. Zangeneh.M, Goto.A, Takemura T. Suppression of secondary flows in a mixed -flow pump impeller by application of three-dimensional inverse design method: part1: design and numerical validation [J]. ASME Journal of Turbo-machinery, 118, 536(1996)

8. Jin Shuanbao, Wang Yongsheng, Ding Jiangming. Three-dimensional design and numerical experiment of mixed-flow waterjet with $\mathrm{CFD}[\mathrm{J}]$. Journal of Harbin Engineering University, 33,1223(2012) 